\title{
FROM THE DESK OF EDITOR IN CHIEF
}

\section{From the Desk of Editor in Chief}

I am very happy that the volume-8, No-1, issue of Anwer Khan Modern Medical College Journal has published in time. It is very encouraging to note that due to tremendous support \& interest amongst our readers there is increased number of submitted articles for publication in AKMC journal. For this reason, we have increased the volume of present issue comprising of editorial, nine original articles, one review article $\&$ four case reports.

Editorial on "Changing Faces of Tissue Diagnosis in the Present Era" is a timely subject focussing on different aspects of tissue diagnosis including PCR, FISH, MLPA, Micro-array technology, NGS. These will aware the physicians about the importance of these newer techniques for tissue diagnosis.

Original article on "Pattern of Histopathologically Confirmed Uterine \& Ovarian Anommeli among 98 Hysterecomies Attending a Private Medical College Hospital, Dhaka" highlighted the importance of histopathological examination of all hysterectomy samples.

Original article on "A Retrospective Analysis of Efficacy \& Toxicity of Bevacizumab in Combination with Chemotherapy for the Management of NSCLC in Bangladeshi Population" emphasises the importance of combination chemotherapy \& addition of Bevacizumab as a targeted therapy for better management of NSCLC.

Article on "CTscan Findings of Orbital Mass Among Paediatric Patients at a Territory Care Hospital in Bangladesh" identified that sensitivity of diagnosis of orbital tumour by CT-scan is very high about $93.3 \%$ alongside histopathological diagnosis.

Original article on "Antinociceptive and anti inflammatory effects of combined administratin of $\alpha-$ tocopherol and Morphine in Long Evans Rats" conlcuded that combined administration of Morphine Sulphate and $\alpha$-tocopherol are more effective in lowering pain \& inflammation than individual administration of morphine sulphate.

Article on "Comparative Study of Protective Effect of Tomato Juice \& Hexane Extract of Tomato" found both have protective effect against hapyerlipidemia \& oxidative stress and tomato can be recommended in our daily food list.

Article "On Acute Kidney Injury Patients of Intensive Care Unit" conluced that the incidence of AKI is higher in patients admitted to ICU, which is associated with poor outcome \& reduced survival.

Article on "Administration of Testosterone \& its Rationale in the Treatment of Erectile Dysfunction" AKMMC J 2017; 8(1) : 79-79 found that testosterone replacement therapy is beneficial in patients with ED due to hypogonadism.

Article on "Role of Combined Mibepristone \& Misoprostol Versus Misoprostol Alone in Induction of Labour in Patients with Interauterine Foetal Death a Randomized Comparison Between Their Outcomes" found that combined miberpristone \& misoprostol are more effective in patients with intrautcrine foetal death for induction of labour.

The last original article on "Prevalence of NCDS among rural households of Dhamrai upazila, Dhaka" concluded that proportion of NCDs among the rural households are alarming \& people are suffering from various comorbidities along with NCDS. The study findings demand that better strategic plan for surveillance \& prevention of NCDs are a must.

Review article on "Reasons of Absenteeism Among Undergraduate, Medical Students." emphasized the need for early detection \& prevention of dreadful comequences of absenteeism of medical students on academic performances. The identified causes are lack of subject matter interest, poor teaching strategies, unfavorable learning environment, ill-health, sleeplessness \& poor relations with lectures. The medical college authority should look into it.

First case report on "successful manamgent of peripartum cardiomyopathy" emphasizes the need of echocardiogram for early diagnosis \& management \& to reduce the mortality.

Second case report on "Osteoma left Mastoid Bone" Report a Case Report of Rare Benign Bone tumour in a rare location.

Third case report on "Perforated Appendicitis with Appendicular Abscess" emphasizes the Need of early diagnosis \& management of acute appendicitis to prevent its dreadful complications.

Last case report on "Blunt Right ICA Baffle grade IV injury in a case of polytrauma: Successfully managed by Decompressive Craniotmy" stated the importance of early diagnosis \& management of RTA polytrauma leading to TBI by clinical, neurological \& CT-scan angiogram.

Lastly I hope the readers will find this issue an interesting one \& give their valuable feedback for improvement in future.

Prof. Dr. Md. Tahminur Rahman

Editor in Chief, AKMMC Journal

Email: mtahminur@yahoo.com

Phone: 008801817561519 